\title{
Diagnostic and prognostic role of neutrophil/lymphocyte ratio, platelet/lymphocyte ratio, and lymphocyte/monocyte ratio in patients with osteosarcoma
}

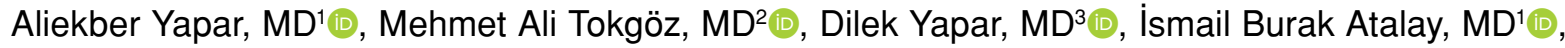 \\ Coşkun Ulucaköy, MD¹D, Bedii Şafak Güngör, $\mathrm{MD}^{1}$ (iD \\ 'Department of Orthopedics and Traumatology, Ankara Oncology Training and Research Hospital, Ankara, Turkey \\ ${ }^{2}$ Department of Orthopaedics and Traumatology, Dr. Nafiz Korez Sincan State Hospital, Ankara, Turkey \\ ${ }^{3}$ Department of Public Health, Gazi University Faculty of Medicine, Ankara, Turkey
}

Osteosarcoma is the most common primary malignant bone malignancy which accounts for about twothirds of all cases..$^{[1,2]}$ Most of the osteosarcomas are classified as traditional type, high-grade tumors, and diagnosed by radiological examinations and biopsy. Typical osteosarcomas are osteoblastic or osteolytic lesions which can be demonstrated with a periosteal reaction on conventional radiographs. However, osteosarcoma can break out beyond the traditional types of findings, and diagnosing some of its variants is difficult for clinicians. ${ }^{[3]}$ Serum tumor markers used in the diagnosis of osteosarcoma usually result in a very little success. While the response to treatment and disease prognosis can be estimated with the help

Received: October 28, 2020

Accepted: December 11, 2020

Published online: June 11, 2021

Correspondence: Coskun Ulucaköy, MD. SBÜ Dr. Abdurrahman Yurtaslan Onkoloji Eğitim ve Araştırma Hastanesi Ortopedi ve Travmatoloji Kliniği, 06105 Yenimahalle, Ankara, Türkiye.

E-mail: coskunulucakoy@gmail.com

Doi: 10.52312/jdrs.2021.79775

Citation: Yapar A, Tokgöz MA, Yapar D, Atalay IB, Ulucaköy C, Güngör BŞ. Diagnostic and prognostic role of neutrophil/ lymphocyte ratio, platelet/lymphocyte ratio, and lymphocyte/ monocyte ratio in patients with osteosarcoma. Jt Dis Relat Surg 2021;32(2):489-496.

(02021 All right reserved by the Turkish Joint Diseases Foundation

This is an open access article under the terms of the Creative Commons Attribution-NonCommercial License, which permits use, distribution and reproduction in any medium, provided the original work is properly cited and is not used for commercial purposes (http://creativecommons.org/licenses/by-nc/4.0/).

\section{ABSTRACT}

Objectives: This study aims to evaluate the diagnostic and prognostic significance of neutrophil-to-lymphocyte ratio (NLR), platelet-to-lymphocyte ratio (PLR), and lymphocyte-to-monocyte ratio (LMR) values in patients with osteosarcoma.

Patients and methods: A total of 172 patients (111 males, 61 females; mean age: $24.3 \pm 15.3$ years; range, 7 to 82 years) diagnosed with osteosarcoma in our institution between January 2002 and December 2018 were retrospectively analyzed. A total of 165 healthy individuals (115 males, 50 females; mean age: $20.2 \pm 9.2$ years; range, 10 to 65 years) who did not have infectious, rheumatological or hematological diseases or any pathological finding were assigned as the control group. The clinical, laboratory, and demographic findings of the patients were obtained from hospital records. Pre-treatment NLR, PLR, and LMR values were calculated in all patients. Diagnostic and prognostic values of pre-treatment NLR, PLR and LMR were assessed using receiver operating curve (ROC) analysis. The Kaplan-Meier method was used for survival analysis.

Results: For diagnostic approach, the highest significance in area under the curve (AUC) values was obtained for NLR (AUC=0.763). The AUC for PLR and LMR was statistically significant, while the statistical power was weak compared to NLR (AUC=0.681 and 0.603). The NLR, PLR, and LMR were found to be predictors of mortality. The cut-off value was found to be 3.28 for NLR, 128 for PLR, and 4.22 for LMR. The prognostic value of NLR for mortality was higher than (AUC=0.749) PLR (AUC $=0.688)$ and LMR (AUC=0.609). The NLR, PLR, and LMR were associated with overall survival (OS). There was a significant difference in the median OS time among the NLR, PLR, and LMR values (log-rank test order $\mathrm{p}<0.001$, $\mathrm{p}=0.001$, and $\mathrm{p}=0.004$, respectively).

Conclusion: Based on our study results, pre-treatment NLR, PLR and MLR have diagnostic and prognostic values in osteosarcoma.

Keywords: Diagnostic significance, neutrophil/lymphocyte ratio, osteosarcoma, prognostic significance. 
of some serum markers, there is no inexpensive and widely available marker to help in diagnosis. ${ }^{[4]}$ Besides, poor prognosis and high mortality rates in metastatic osteosarcoma are similar to those of 20 years ago, despite current multimodality treatment. ${ }^{[5]}$

An increasing number of evidence has shown that, in cancer patients, host-related variables may help in diagnosis with an impact on prognosis. In particular, systemic inflammatory response and related variables are reported to have a remarkable effect. ${ }^{[6,7]}$ Demonstration of the increased presence of inflammatory cells in the tumor local microenvironment has also triggered the interest in markers, such as circulating leukocyte counts and neutrophil-to-lymphocyte ratio (NLR). ${ }^{[8]}$ It has been shown that inflammation-based scores may contribute to diagnostic and prognostic evaluation in various types of cancer. ${ }^{[9-12]}$

In the literature, prognostic markers calculated on the basis of inflammatory cells are shown to have a prognostic value in osteosarcoma patients. However, both the diagnostic and prognostic effects of these markers on osteosarcoma have not been fully clarified. In the present study, we aimed to investigate the effect of prognosis of the levels of pre-treatment inflammatory markers (NLR, plateletto-lymphocyte ratio [PLR], and lymphocyte-tomonocyte ratio [LMR]) on patients with osteosarcoma and to compare them with the healthy controls for identifying the diagnostic value.

\section{PATIENTS AND METHODS}

This single-center, retrospective study was conducted at Dr. Abdurrahman Yurtaslan Ankara Oncology Training and Research Hospital, Department of Orthopedics and Traumatology, between January 2002 and December 2018. A total of 172 patients (111 males, 61 females; mean age: $24.3 \pm 15.3$ years; range, 7 to 82 years) diagnosed with osteosarcoma in our institution were included. A total of 165 healthy individuals (115 males, 50 females; mean age: $20.2 \pm 9.2$ years; range, 10 to 65 year) who did not have infectious, rheumatological or hematological diseases or any pathological finding were assigned as the control group. The control group who referred to the outpatient clinic of orthopedic and traumatology with non-specific symptoms was similar in terms of age and sex to the patient group. A written informed consent was obtained from each participant and/or his/her legal guardian. The study protocol was approved by the Dr. Abdurrahman Yurtaslan Ankara Oncology Training and Research Hospital Ethics Committee (date/no: 2020-06/647). The study was conducted in accordance with the principles of the Declaration of Helsinki.

Patients with a histopathologically confirmed osteosarcoma diagnosis who had no previous anticancer treatment and who had complete medical and follow-up records were included. Patients with elevated C-reactive protein results, who were lost to follow-up, those with diabetes mellitus, infections disease, rheumatological diseases and other inflammatory diseases, any hematological disease, previously treated with non-steroid antiinflammatory drugs, and those with missing medical records were excluded from the study. Clinical and demographic data of the patients were reviewed from the hospital records. Age, sex, tumor location, side, pre-treatment complete blood count results, date of diagnosis, the final follow-up date, surgical procedure, the status of metastasis at diagnosis, mortality, and recurrence records were reviewed from the patient files. The NLR and PLR were calculated as the absolute count of neutrophils and platelets, respectively, divided by the absolute lymphocyte count. The LMR was calculated as the absolute count of lymphocyte divided by the absolute monocyte count.

\section{Statistical analysis}

Statistical analysis was performed using the IBM SPSS for Windows, version 22.0 software (IBM Corp., Armonk, NY, USA). Descriptive statistics are presented in number and percentage for categorical variables and in mean \pm standard deviation or median (min-max) for continuous variables. Normal distribution for continuous variables were assessed with visual (histograms and probability graphics) and analytic methods (Kolmogorov-Smirnov test). In the data that do not fit the normal distribution, the Mann-Whitney $\mathrm{U}$ test was used for comparison analysis between the two independent groups. Comparison analyses for categorical variables between independent groups were done by chi-square test. Diagnostic and prognostic values of pre-treatment NLR, PLR, and LMR were assessed using the receiver operating curve (ROC) analysis. The area under the ROC curve (AUC) results were considered excellent for AUC values between 0.9-1, good for AUC values between 0.8-0.9, fair for AUC values between 0.7-0.8, poor for AUC values between 0.6-0.7, and failed for AUC values between 0.5-0.6. ${ }^{[13,14]}$ Following the ROC analysis results, the AUC and cut-off values, sensitivity and specificity of these cut-offs, likelihood ratio (LHR), positive predictive 


\begin{tabular}{|c|c|c|}
\hline \multicolumn{3}{|c|}{$\begin{array}{l}\text { Baseline demographic characteristics of patients with } \\
\text { osteosarcoma }(n=172)\end{array}$} \\
\hline Characteristic & $\mathrm{n}$ & $\%$ \\
\hline \multicolumn{3}{|l|}{ Localization } \\
\hline Femur & 98 & 57.0 \\
\hline Tibia & 30 & 17.4 \\
\hline Humerus & 16 & 9.3 \\
\hline Fibula & 13 & 7.6 \\
\hline Pelvis & 12 & 7.0 \\
\hline Shoulder & 1 & 0.6 \\
\hline Scapula & 1 & 0.6 \\
\hline Radius & 1 & 0.6 \\
\hline \multicolumn{3}{|c|}{ Metastasis at diagnosis } \\
\hline No & 152 & 88.4 \\
\hline Yes & 20 & 11.6 \\
\hline \multicolumn{3}{|l|}{ Mortality } \\
\hline Non-survivors & 76 & 44.2 \\
\hline Survivors & 96 & 55.8 \\
\hline
\end{tabular}

value (PPV) and negative predictive value (NPV) were calculated. Survival analyses were performed using the Kaplan-Meier method and log-rank test. A $p$ value of $<0.05$ was considered statistically significant.

\section{RESULTS}

Of the patients with osteosarcoma, $11.6 \%$ of the patients had metastases at the time of diagnosis, while $44.2 \%$ of the patients died during follow-up. According to the localization, tumors were mostly located in the femur (57\%) and tibia (17.4\%). The age and sex distributions of the control and the patient groups were similar $(\mathrm{p}=0.528$ and $\mathrm{p}=0.313$, respectively) (Table I).
There was no significant correlation between age and NLR $(\mathrm{r}=0.052, \mathrm{p}=0.337)$, PLR $(\mathrm{r}=0.054, \mathrm{p}=0.320)$ and LMR ( $\mathrm{r}=0.046, \mathrm{p}=0.396)$. The NLR $(\mathrm{p}=0.544)$, PLR $(p=0.796)$ and LMR $(p=0.364)$ between the age groups of $\leq 18$ years old and $>18$ years old were similar. When it was evaluated separately for patient and control groups, each NLR ( $\mathrm{p}_{\text {control }}=0.938$, $\mathrm{p}_{\text {patients }}=0.243$ ), PLR ( $\left.\mathrm{p}_{\text {control }}=0.504, \mathrm{p}_{\text {patients }}=0.282\right)$, and LMR $\left(\mathrm{p}_{\text {control }}=0.658\right.$, $\left.p_{\text {patients }}=0.355\right)$ among age groups of $\leq 18$ years old and $>18$ years old were found to be similar.

The NLR and PLR values of the sarcoma group were significantly higher than the controls $(p<0.001)$ (Table II). The LMR values were statistically significantly lower in the sarcoma group, compared to the controls $(p=0.001)$. The diagnostic power of NLR, PLR, and LMR with ROC analysis was evaluated (Figure 1). Accordingly, the highest significance in AUC values was obtained for NLR (AUC $=0.763$ ). The calculated value of AUC for PLR and LMR was statistically significant, while the statistical power was weak, compared to NLR (AUC=0.681 and 0.603).

The cut-off values were determined for all three parameters, and sensitivity, specificity, + LHR, PPV, and NPV values of the cut-off values were calculated. The predictability of NLR values of $\geq 2.45$ in the diagnostic approach for osteosarcoma was higher than the other two parameters (sensitivity $=64.5 \%$, specificity $=78.8 \%$, + LHR $=3.04$, $\mathrm{PPV}=75 \%$, and NPV=67.7) (Table III). The cut-off value for PLR was found to be 119 (sensitivity $=60.5 \%$, specificity $=64.2 \%,+\mathrm{LHR}=1.69, \mathrm{PPV}=63.4 \%$, and $\mathrm{NPV}=60.7)$ for diagnostic approach.

The predictability of NLR, PLR, and LMR values for mortality was evaluated by the ROC analysis and the statistical significance of AUC values obtained from ROC analysis was presented in Table IV and Figure 2. The NLR, PLR, and LMR were found to be

\begin{tabular}{|c|c|c|c|c|c|c|c|c|c|}
\hline \multicolumn{10}{|c|}{$\begin{array}{l}\text { TABLE II } \\
\text { Comparison of patients and control groups }(n=337)\end{array}$} \\
\hline & \multicolumn{4}{|c|}{ Controls $(n=165)$} & \multicolumn{4}{|c|}{ Patients $(n=172)$} & \multirow[b]{2}{*}{$p$} \\
\hline & $\mathrm{n}$ & $\%$ & Median & Min-Max & $\mathrm{n}$ & $\%$ & Median & Min-Max & \\
\hline Age (year) & & & 19 & $10-65$ & & & 19 & $7-82$ & $0.520^{*}$ \\
\hline Sex & & & & & & & & & $0.313^{* *}$ \\
\hline Male & 115 & 69.7 & & & 111 & 64.5 & & & \\
\hline Female & 50 & 30.3 & & & 61 & 35.5 & & & \\
\hline Neutrophil-to-lymphocyte ratio & & & 1.83 & $0.11-9.18$ & & & 3.33 & $0.46-13.51$ & $<0.001^{*}$ \\
\hline Platelet-to-lymphocyte ratio & & & 110.53 & 63.7-296.7 & & & 127.84 & $40.51-807.8$ & $<0.001^{*}$ \\
\hline Lymphocyte-to-monocyte ratio & & & 4.81 & $1.1-9.13$ & & & 4.19 & $0.66-20.9$ & $0.001^{*}$ \\
\hline
\end{tabular}


(a)

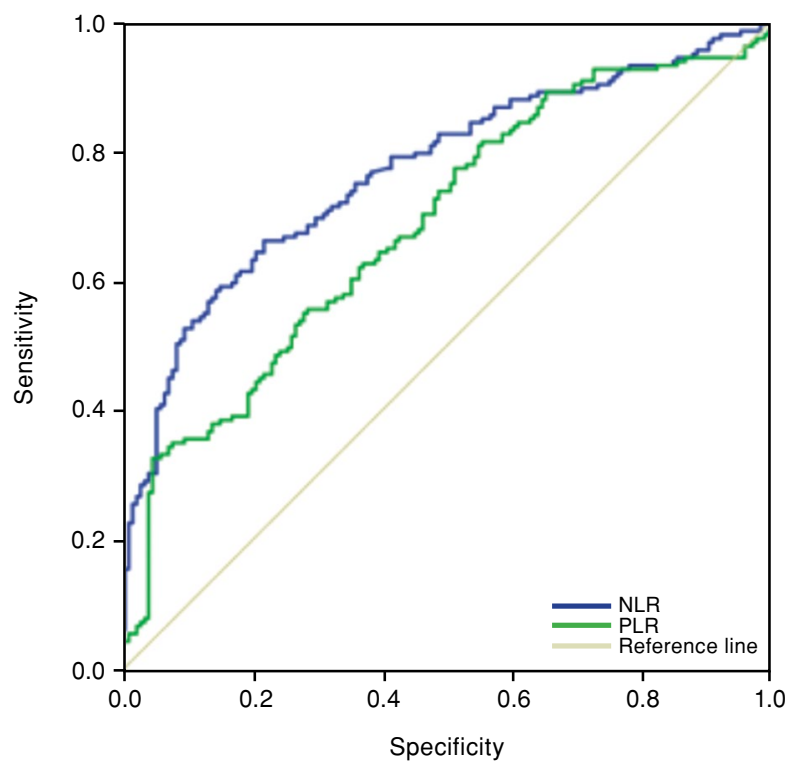

(b)

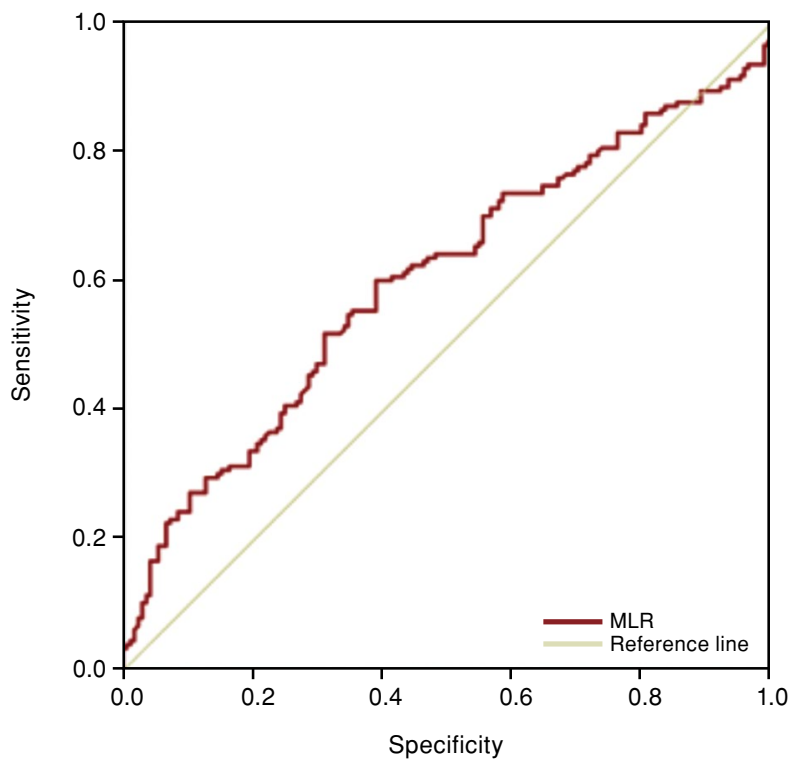

FIGURE 1. Receiver operating characteristic (ROC) curves for diagnostic value of the NLR (a), PLR (a), and LMR (b). (a) Larger results of NLR and PLR indicate more diagnostic positive test for sarcomas. (b) Smaller results of LMR indicate more diagnostic positive test for sarcomas.

NLR: Neutrophil-to-lymphocyte ratio; PLR: Platelet-to-lymphocyte ratio; LMR: Lymphocyte-to-monocyte ratio.

predictors of mortality. The cut-off value was found to be 3.28 for NLR, 128 for PLR, and 4.22 for LMR. The prognostic value of NLR for mortality was higher than $(\mathrm{AUC}=0.749)$ PLR $(\mathrm{AUC}=0.688)$ and LMR $(\mathrm{AUC}=0.609)$.
The median overall survival (OS) duration of the osteosarcoma patients was 46.6 (range, 22.4 to 70.82) months. While the one-year OS rate was $86.2 \%$, the three-year OS rate decreased to $59.7 \%$ and the

\section{TABLE III}

Statistical analysis of various diagnostic approaches for the predictive value of pre-treatment NLR, PLR and LMR ( $\mathrm{n}=337$ )

\begin{tabular}{|c|c|c|c|c|c|c|c|c|}
\hline & $\mathrm{AUC}(95 \% \mathrm{Cl})$ & $p$ & Cut-off & $\begin{array}{c}\text { Sensitivity } \\
(\%)\end{array}$ & $\begin{array}{c}\text { Specificity } \\
(\%)\end{array}$ & + LHR & $\begin{array}{l}\text { PPV } \\
(\%)\end{array}$ & $\begin{array}{c}\text { NPV } \\
(\%)\end{array}$ \\
\hline NLR & $0.763(0.711-0.814)$ & $<0.001$ & $\geq 2.45$ & 64.5 & 78.8 & 3.04 & 75 & 67.7 \\
\hline PLR & $0.681(0.624-0.738)$ & $<0.001$ & $\geq 119$ & 60.5 & 64.2 & 1.69 & 63.4 & 60.7 \\
\hline LMR & $0.603(0.542-0.663)$ & 0.001 & $\leq 4.47$ & 59.9 & 60.6 & 1.54 & 61.3 & 59.2 \\
\hline
\end{tabular}

AUC: Area under the curve; Cl: Confidence interval; +LHR: Positive likelihood ratio; PPV: Positive predictive value; NPV: Negative predictive value; NLR: Neutrophil-tolymphocyte ratio; PLR: Platelet-to-lymphocyte ratio; LMR: Lymphocyte-to-monocyte ratio.

\section{TABLE IV}

Statistical analysis of various prognostic approaches for the predictive value of pre-treatment NLR, PLR and LMR ( $\mathrm{n}=172)$

\begin{tabular}{|c|c|c|c|c|c|c|c|c|}
\hline & $\mathrm{AUC}(95 \% \mathrm{Cl})$ & $p$ & Cut-off & $\begin{array}{c}\text { Sensitivity } \\
(\%)\end{array}$ & $\begin{array}{c}\text { Specificity } \\
(\%)\end{array}$ & +LHR & $\begin{array}{l}\text { PPV } \\
(\%)\end{array}$ & $\begin{array}{l}\text { NPV } \\
(\%)\end{array}$ \\
\hline NLR & $0.749(0.675-0.824)$ & $<0.001$ & $\geq 3.28$ & 61.8 & 76 & 2.58 & 67.1 & 71.6 \\
\hline PLR & $0.688(0.608-0.768)$ & $<0.001$ & $\geq 128$ & 68.4 & 64.6 & 2.01 & 60.5 & 72.1 \\
\hline LMR & $0.609(0.525-0.664)$ & 0.014 & $\leq 4.22$ & 63.2 & 57.3 & 1.47 & 53.9 & 66.3 \\
\hline
\end{tabular}


(a)

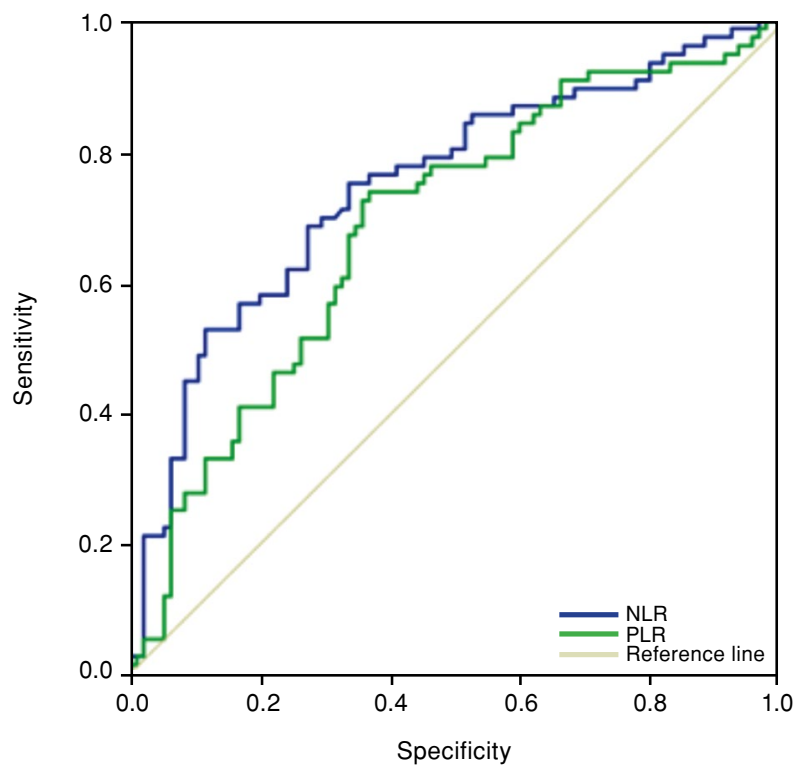

(b)

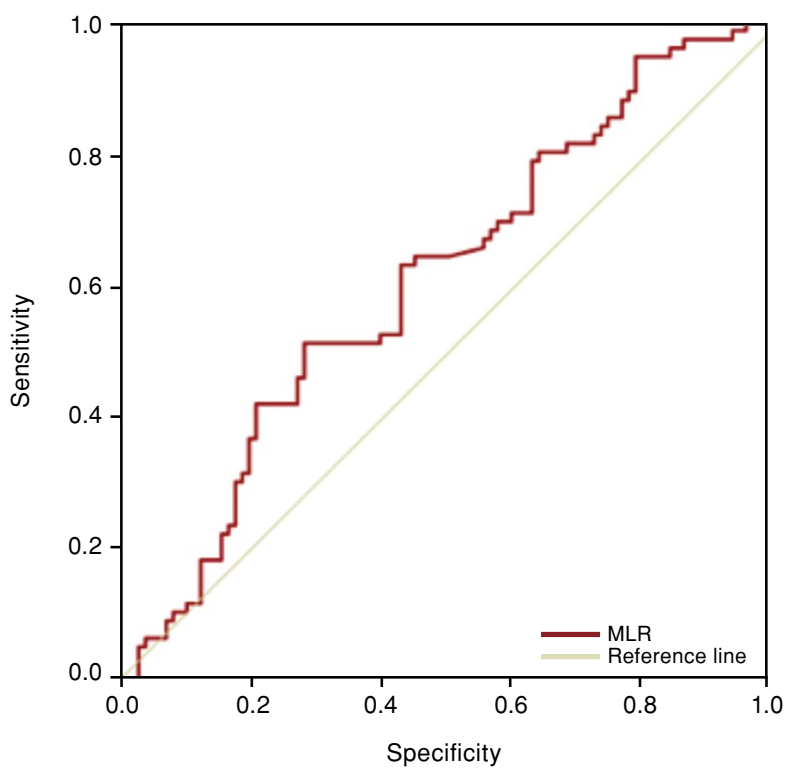

FIGURE 2. Receiver operating characteristic (ROC) curves for prognostic value of the NLR (a), PLR (a), and LMR (b). (a) Larger results of NLR and PLR indicate more prognostic positive test for sarcomas. (b) Smaller results of LMR indicate more diagnostic positive test for sarcomas.

NLR: Neutrophil-to-lymphocyte ratio; PLR: Platelet-to-lymphocyte ratio; LMR: Lymphocyte-to-monocyte ratio.

\begin{tabular}{|c|c|c|c|c|c|c|}
\hline \multicolumn{7}{|c|}{$\begin{array}{c}\text { TABLE V } \\
\text { Overall survival rates }(n=172)\end{array}$} \\
\hline & $\begin{array}{l}\text { Log Rank test } \\
\text { (p) }\end{array}$ & $\begin{array}{l}\text { Overall survival, } \\
\text { months } \\
\text { Median }(95 \% \mathrm{Cl})\end{array}$ & $\begin{array}{c}\text { 1-year } \\
\text { survival rate } \\
(\%)\end{array}$ & $\begin{array}{c}\text { 3-year } \\
\text { survival rate } \\
(\%)\end{array}$ & $\begin{array}{c}\text { 5-year } \\
\text { survival rate } \\
(\%)\end{array}$ & $\begin{array}{c}\text { 10-year } \\
\text { survival rate } \\
(\%)\end{array}$ \\
\hline All patients & - & $46.6(22.4-70.82)$ & 86.2 & 59.7 & 47.2 & 39.0 \\
\hline NLR & $<0.001$ & & & & & \\
\hline$<3.28$ & & 87.3 (62.8-111.7) & 92.3 & 76 & 67.7 & 47.8 \\
\hline$\geq 3.28$ & & $31.7(22.7-40.6)$ & 78.0 & 39.7 & 23 & 35.3 \\
\hline PLR & 0.001 & & & & & \\
\hline$<128$ & & $92.4(74.7-112.9)$ & 89.7 & 73.3 & 65.3 & 43.5 \\
\hline$\geq 128$ & & $36.0(29.2-42.7)$ & 83 & 48.9 & 33.7 & 36.2 \\
\hline LMR & 0.004 & & & & & \\
\hline$\leq 4.22$ & & $73.6(44.1-103.2)$ & 90.8 & 73.2 & 61.6 & 40.2 \\
\hline$>4.22$ & & $33.6(27.8-39.4)$ & 82 & 47.5 & 34.3 & 38.2 \\
\hline
\end{tabular}

10-year OS rate to $39 \%$ (Figure 3a). There was a significant difference in the median OS time according to NLR, PLR, and LMR values (log-rank test order $\mathrm{p}<0.001, \mathrm{p}=0.001$, and $\mathrm{p}=0.004$, respectively) (Table V, Figures 3b-d).

\section{DISCUSSION}

In the current study, we evaluated factors showing the systemic inflammatory response from two different perspectives. Pre-treatment values of NLR, PLR, and LMR in the peripheral blood of 
(a)

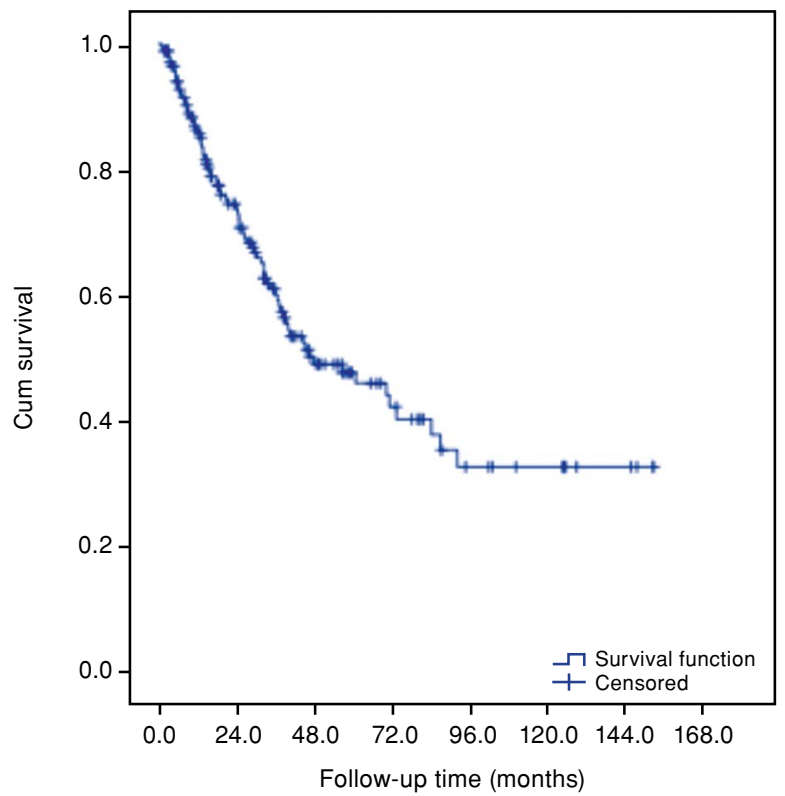

(c)

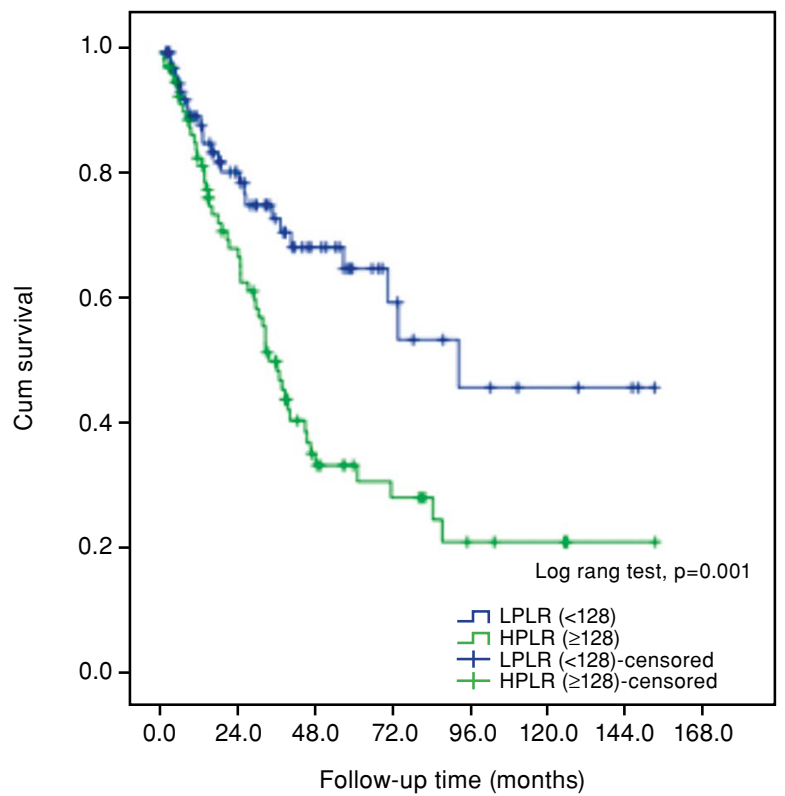

(b)

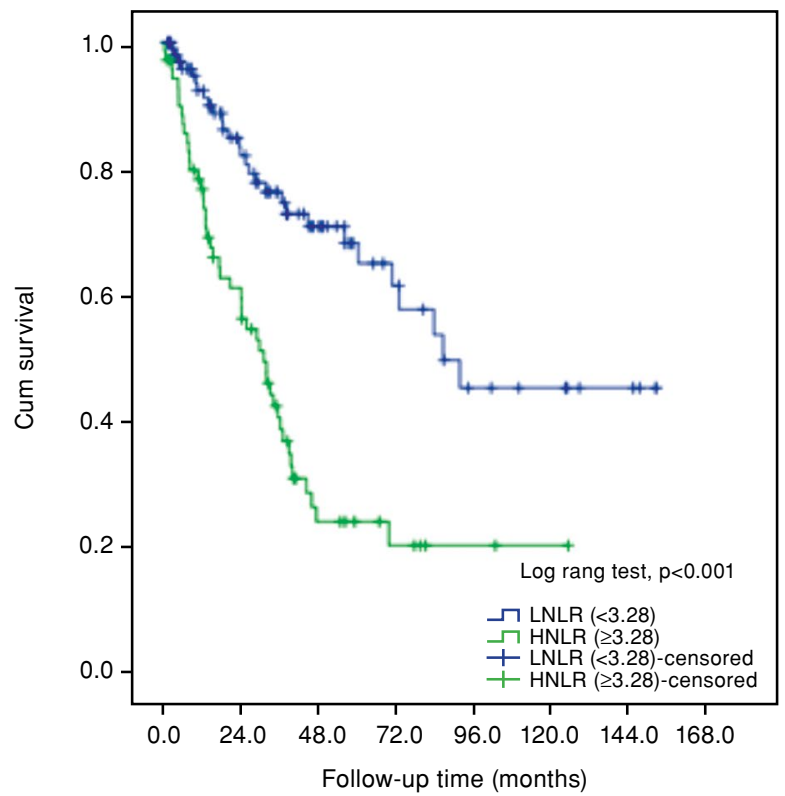

(d)

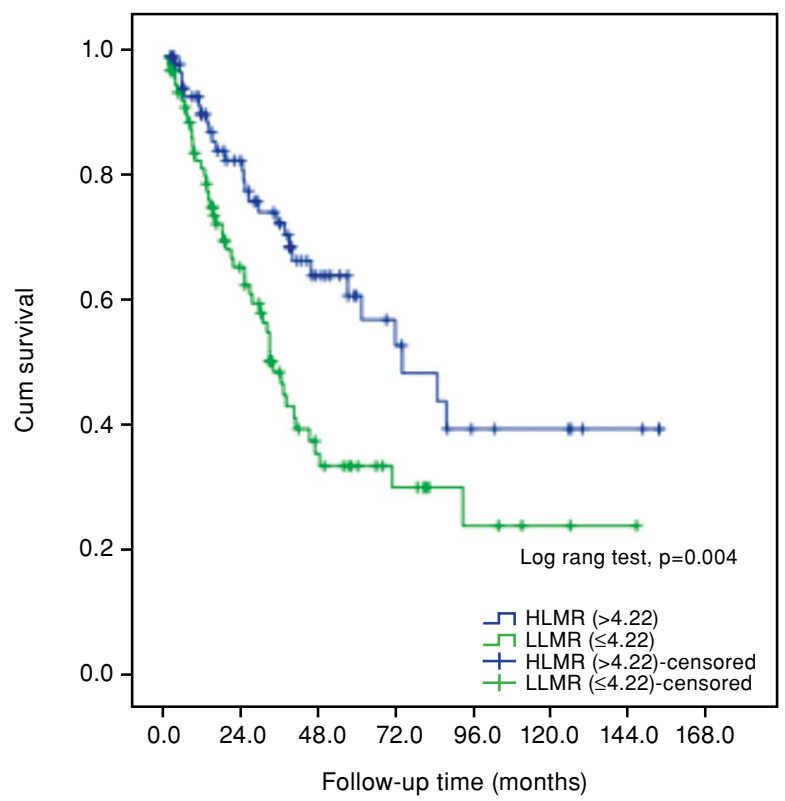

FIGURE 3. Kaplan-Meier curves for overall survival. (a) Overall survival curve and of patients with osteosarcoma. The OS rates of patients at $1,3,5$, and 10 years after surgery were $86.2,59.7,47.2$ and $39 \%$, respectively. (b) Overall survival curve of patients with osteosarcoma in different NLR groups. (c) Overall survival curve of patients with osteosarcoma in different PLR groups. (d) Overall survival curve of patients with osteosarcoma in different LMR groups.

OS: Overall survival; NLR: Neutrophil-to-lymphocyte ratio; LNLR: Low neutrophil-to-lymphocyte ratio; HNLR: High neutrophil-to-lymphocyte ratio; LPLR: Low platelet-to-lymphocyte ratio; HPLR: High platelet-to-lymphocyte ratio; LLMR: Low lymphocyte-to-monocyte ratio; HLMR: High lymphocyteto-monocyte ratio.

osteosarcoma patients were found to be diagnostic and prognostic values. The prognostic value of pretreatment inflammatory markers (NLR, PLR, and LMR) in the osteosarcoma patients was mentioned in the literature. ${ }^{[5,15,16]}$ Consistent with previous studies, our study confirmed that high NLR, PLR, and low LMR were significantly associated with poor OS.

To the best of our knowledge, the current study is the one of the premise studies showing both diagnostic and prognostic value of inflammatory 
based markers. ${ }^{[17]}$ We demonstrated that inflammatory markers, particularly NLR, could be significantly supportive in diagnosing osteosarcoma. In a patient having a suspicion of osteosarcoma, the clinical suspicion increased, when the NLR value was $\geq 2.45$, while the probability of the disease progressing with poor prognosis became stronger, if the pre-treatment NLR was $\geq 3.28$.

In a study, the NLR cut-off values were found to be 3.43 for OS and 3.67 for progression-free survival, and it was found that the probability of poor prognosis on both parameters increased in patients with NLR values above the cut-off. ${ }^{[18]}$ The prognostic value calculations in the current study are consistent with the literature. Another important finding of the current study is that the OS rate of patients with an NLR value of $\geq 3.28$ was significantly lower at three $(39.7 \%)$ and five $(23 \%)$ years.

Similar to changes in peripheral blood immune cells, changes in immune infiltrations around the tumor microenvironment are also reliable and effective prognostic factors for many tumors, including bone and soft tissue sarcomas. ${ }^{[19]}$ Besides, early peripheral blood lymphocyte recovery after initiation of chemotherapy is a reliable prognostic indicator for superior outcomes in patients with osteosarcoma. ${ }^{[2,21]}$ Considering the aforementioned information and current study findings, the high NLR, PLR, and low LMR ratio appeared as a poor prognostic indicator, which could be due to reflection of the inability of the host metabolism to respond to the tumor with sufficient cellular immunity.

In the literature, there are studies on the diagnostic value of inflammatory variables in malignancies. In a study, the NLR was shown to have a diagnostic value for cartilaginous tumors, although it was not found to be effective in distinguishing the subtypes of the tumor. ${ }^{[22]}$ Another study demonstrated that NLR + LMR combination was effective both in separating glioma from acoustic neuroma and meningioma, and differential diagnosis of glioblastoma multiforme from low-grade gliomas. ${ }^{[9]}$ The value of inflammatory markers was also investigated in determining the malignancy potential of spinal tumors and the cutoff value was calculated as 3.19 for NLR and as 141 for PLR value. Malignancy potential of spinal mass patients with the results above these values has been reported to be high. ${ }^{[23]}$ Although there are studies showing the diagnostic value of inflammatory markers, a study on ovarian mass reported that NLR was an ineffective marker in predicting the malignant characteristics of a pelvic mass. ${ }^{[2]]}$ The findings of the current study showed that inflammatory variables could be supportive in diagnosing osteosarcoma such as spinal masses, cartilaginous tumors, and gliomas.

Nonetheless, there are several limitations to this study. First, this study has a single-center, retrospective design. Second, despite its advantages in this cohort, inflammatory markers were a non-specific predictor for osteosarcoma and, thus, inevitably had intrinsic weaknesses and limitations. However, there are no specific serum markers for osteosarcoma. Non-specific findings such as NLR, PLR, and LMR could be beneficial to support the suspicion of clinical and radiological osteosarcoma for some patients.

On the other hand, the current study has several strengths. First, the infection markers were evaluated and the effects of the infection on the inflammatory markers were prevented by excluding the patients who were considered to be infected. Second, both diagnostic and prognostic values of inflammatory markers were evaluated and cut-off values were separately specified for both. In the current study, the clinician should also pay attention to the pre-treatment high NLR to evaluate the OS in bone masses with suspected malignancy. The NLR, PLR, and LMR are simple, low cost, easy to measure with widely used and standardized tests, and the current study is the first to demonstrate the diagnostic value of these nonspecific inflammatory markers.

In conclusion, our study results suggest that patients with a bone mass with malignant potential have higher levels of NLR and PLR and lower levels of LMR than healthy individuals. Pre-treatment NLR, PLR, and MLR have diagnostic and prognostic values in osteosarcoma, supporting the prognostic value of inflammatory markers in osteosarcoma. In addition, NLR may be a superior distinguishing factor to LMR and PLR. There are many different situations which determine the prognosis of neoplastic diseases, and NLR may be one of them. However, the NLR values cannot be a standalone decision maker in patients with osteosarcoma, but they can be one of the variables to be taken into account while making a treatment plan. Further multi-center, large-scale, prospective studies are needed to confirm our findings and investigate the value of the combined use of these inflammatory markers to prolong the remission time of patients with osteosarcoma.

\section{Declaration of conflicting interests}

The authors declared no conflicts of interest with respect to the authorship and/or publication of this article.

\section{Funding}

The authors received no financial support for the research and/or authorship of this article. 


\section{REFERENCES}

1. Ferguson JL, Turner SP. Bone cancer: diagnosis and treatment principles. Am Fam Physician 2018;98:205-13.

2. Ritter J, Bielack SS. Osteosarcoma. Ann Oncol 2010;21 Suppl 7:vii320-5.

3. Hayashi K, Tsuchiya H, Yamamoto N, Shirai T, Nishida H, Takeuchi A, et al. Diagnosis and treatment of low-grade osteosarcoma: experience with nine cases. Int J Clin Oncol 2014;19:731-8.

4. Savitskaya YA, Rico-Martínez G, Linares-González LM, Delgado-Cedillo EA, Téllez-Gastelum R, Alfaro-Rodríguez $\mathrm{AB}$, et al. Serum tumor markers in pediatric osteosarcoma: a summary review. Clin Sarcoma Res 2012;2:9.

5. Wang S, Zheng S, Hu K, Sun H, Zhang J, Rong G, et al. A predictive model to estimate the pretest probability of metastasis in patients with osteosarcoma. Medicine (Baltimore) 2017;96:e5909.

6. McMillan DC. Systemic inflammation, nutritional status and survival in patients with cancer. Curr Opin Clin Nutr Metab Care 2009;12:223-6.

7. Roxburgh CS, McMillan DC. Role of systemic inflammatory response in predicting survival in patients with primary operable cancer. Future Oncol 2010;6:149-63.

8. Sorbye SW, Kilvaer TK, Valkov A, Donnem T, Smeland E, Al-Shibli K, et al. Prognostic impact of peritumoral lymphocyte infiltration in soft tissue sarcomas. BMC Clin Pathol 2012;12:5.

9. Zheng SH, Huang JL, Chen M, Wang BL, Ou QS, Huang SY. Diagnostic value of preoperative inflammatory markers in patients with glioma: a multicenter cohort study. J Neurosurg 2018;129:583-92.

10. Idowu OK, Ding $Q$, Taktak AF, Chandrasekar CR, Yin Q. Clinical implication of pretreatment neutrophil to lymphocyte ratio in soft tissue sarcoma. Biomarkers 2012;17:539-44.

11. Nikolić I, Kukulj S, Samaržija M, Jeleč V, Žarak M, Orehovec B, et al. Neutrophil-to-lymphocyte and plateletto-lymphocyte ratio help identify patients with lung cancer, but do not differentiate between lung cancer subtypes. Croat Med J 2016;57:287-92.

12. Sierzega M, Lenart M, Rutkowska M, Surman M, Mytar B, Matyja A, et al. Preoperative neutrophil-lymphocyte and lymphocyte-monocyte ratios reflect immune cell population rearrangement in resectable pancreatic cancer. Ann Surg Oncol 2017;24:808-15.
13. Metz CE. Basic principles of ROC analysis. Semin Nucl Med 1978;8:283-98.

14. Obuchowski NA. Receiver operating characteristic curves and their use in radiology. Radiology 2003;229:3-8.

15. Liu T, Fang XC, Ding Z, Sun ZG, Sun LM, Wang YL. Preoperative lymphocyte-to-monocyte ratio as a predictor of overall survival in patients suffering from osteosarcoma. FEBS Open Bio 2015;5:682-7.

16. Yang S, Wu C, Wang L, Shan D, Chen B. Pretreatment inflammatory indexes as prognostic predictors for survival in osteosarcoma patients. Int J Clin Exp Pathol 2020;13:515-24.

17. Atik OŞ. Is there something new and interesting in my article? Eklem Hastalik Cerrahisi 2019;30:69.

18. Xia WK, Liu ZL, Shen D, Lin QF, Su J, Mao WD. Prognostic performance of pre-treatment NLR and PLR in patients suffering from osteosarcoma. World J Surg Oncol 2016;14:127.

19. Buddingh EP, Kuijjer ML, Duim RA, Bürger H, Agelopoulos $\mathrm{K}$, Myklebost $\mathrm{O}$, et al. Tumor-infiltrating macrophages are associated with metastasis suppression in high-grade osteosarcoma: a rationale for treatment with macrophage activating agents. Clin Cancer Res 2011;17:2110-9.

20. Yang Q, Chen T, Yao Z, Zhang X. Prognostic value of pretreatment Naples prognostic score (NPS) in patients with osteosarcoma. World J Surg Oncol 2020;18:24.

21. Moore C, Eslin D, Levy A, Roberson J, Giusti V, Sutphin R. Prognostic significance of early lymphocyte recovery in pediatric osteosarcoma. Pediatr Blood Cancer 2010;55:1096-102.

22. Yapar A, Ulucaköy C, Sezgin EA, Atalay İB, Ekşioğlu MF. Diagnostic role of neutrophil-to-lymphocyte ratio and monocyte-to-lymphocyte ratio in patients with enchondroma and low-grade chondrosarcoma. Jt Dis Relat Surg 2020;31:286-90.

23. Li Y, Wang B, Zhou S, Jiang L, Yang S, Liu X, et al. Do routine blood test results help in the diagnosis of spine tumors? A retrospective study of the significance of pretreatment neutrophil-to-lymphocyte and platelet-tolymphocyte ratios from 503 spine tumor patients. Medicine (Baltimore) 2019;98:e14902.

24. Topcu HO, Guzel AI, Ozer I, Kokanali MK, Gokturk U, Muftuoglu KH, et al. Comparison of neutrophil/lymphocyte and platelet/ lymphocyte ratios for predicting malignant potential of suspicious ovarian masses in gynecology practice. Asian Pac J Cancer Prev 2014;15:6239-41. 